\section{A case of necrobacillosis}

\author{
A J Chippindale, B Patel, H Mamtora
}

\begin{abstract}
A case of empyema due to Fusobacterium necrophorum infection is described. A history of sore throat followed by metastatic abscesses may alert the clinician to the possibility of this unusual infection.
\end{abstract}

Anaerobic organisms constitute the predominant normal flora of the skin, mucous membranes, and colon but community acquired anaerobic infections are distinctly uncommon in previously healthy people. We report a patient with a severe septicaemic illness due to infection with the anaerobic organism Fusobacterium necrophorum, which resulted in empyema.

\section{Case report}

A 17 year old boy was admitted with a two week history of cough, chest pain, and shortness of breath. The illness began with a sore throat, which had settled before admission, and coryza. Two days before admission his condition deteriorated, with right sided pleuritic chest pain and increased dyspnoea. He was a non-smoker and had been previously well, working as a storeman. He had a history of penicillin allergy.

At admission the patient was unwell, with a fever of $39.4{ }^{\circ} \mathrm{C}$. There were signs of a right sided pneumothorax. The white blood count was $17 \cdot 2 \times 10^{9} / 1$. A chest radiograph confirmed the pneumothorax, and showed an air-fluid level in the right costophrenic sulcus.

Antibiotic treatment with erythromycin was started. The right lung rapidly re-expanded after insertion of an anterior chest drain. The patient, however, remained unwell with persistent cough, fever, and leucocytosis. Serial chest radiographs showed progressive right sided pleural effusion. Eight days after admission a Gram negative anaerobic rod was isolated from a pleural aspirate, though serial blood cultures remained negative. Metronidazole, to which the organism was sensitive, was introduced. The organism was subsequently identified as Fusobacterium necrophorum.

Despite antibiotic treatment the patient's condition deteriorated. The development of multiple fluid levels in the increasingly large right sided empyema (figure) confirmed active anaerobic infection. Aspiration of thick pus from the pleural cavity was only partially successful so the patient underwent thoracotomy. He recovered completely after decortication with continuing antibiotic treatment.

\section{Discussion}

Necrobacillosis, or postanginal septicaemia, is an oropharyngeal infection complicated by local thrombophlebitis and metastatic infection. ${ }^{1}$ The syndrome was considered to be so characteristic that the diagnosis could be suspected on clinical grounds alone. The most frequent bacterial isolate was Bacillus funduliformis, now known as $F$ necrophorum.

Anaerobic infection is uncommon in fit people and is generally associated with recent surgery, antibiotic treatment, diabetes mellitus, or alcoholism. Anaerobic lung infection is often associated with dysphagia and states of impaired consciousness. In most anaerobic infections a mixed bacterial flora is present; the synergistic activity of several species of organisms is required for pathogenicity. Necrobacillosis, unlike other anaerobic infections, induces acute septicaemia in previously healthy young people, $F$ necrophorum being either the sole pathogen or accompanied only by microaerophilic streptococci.

$F$ necrophorum, an anaerobic, non-sporulating, Gram negative rod, is a normal inhabitant of the oral cavity, the female genital tract, and to a lesser extent the gastrointestinal tract. It is associated with infections of the middle ear, paranasal sinuses, mouth, and pharynx. Pharyngeal infections are commonly accompanied by painful lymphadenopathy. In $F$ necrophorum septicaemia pharyngeal infection is often present and is presumed to be the source of the systemic infection. ${ }^{23}$

Manifestations of metastatic infection include septic thrombophlebitis, disseminated intravascular coagulation, abscess formation, peritonitis, enteric and pulmonary fistulas, septic arthritis, osteomyelitis, endocarditis, and meningitis. ${ }^{24}$ Of these, diffuse lung infection is the most frequent and is accompanied by cavitation. ${ }^{35}$ Empyema ${ }^{1}$ and pyopneumothorax are also common. ${ }^{2}$ Jaundice is frequently present.

The isolation of $F$ necrophorum from blood, pleural aspirate or pus is technically demanding, requiring strictly anaerobic conditions and prolonged incubation. Isolation from sputum is unhelpful, as the organism is part of the normal oral flora. It may be readily mistaken

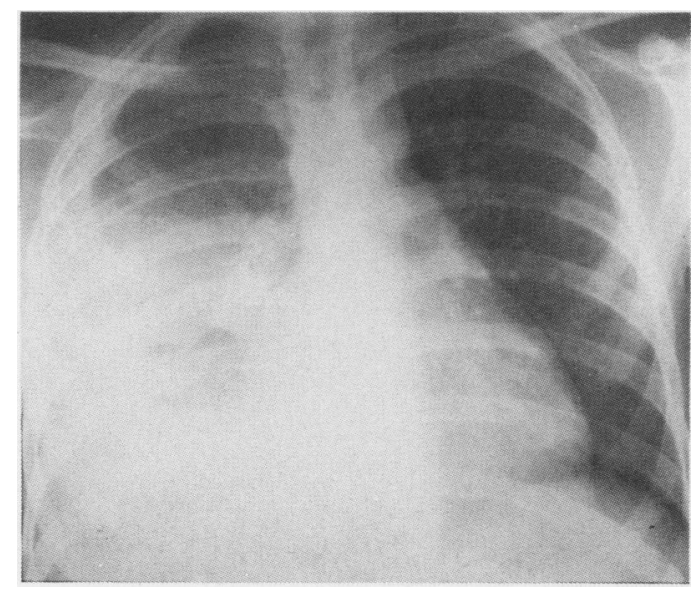

Chest radiograph showing multiple small gas-fluid levels lateral to the right border of the heart. 
for Bacteroides, although the distinctive morphology should alert the microscopist to its true identity. ${ }^{6}$ Gas-liquid chromatography identifies the organism from characteristic volatile fatty acid byproducts.

$F$ necrophorum is especially sensitive to penicillin in vitro and also to cephalosporins, minocycline, metronidazole, and chloramphenicol. ${ }^{7}$ The optimal therapeutic regimen is not known. Response is characteristically slow, and signs may progress despite appropriate antibiotics. ${ }^{8}$ Antibiotics may be necessary for a month or more, and surgical drainage is often required. Mortality remains high.

$F$ necrophorum accounts for only occasional cases of septicaemia. The characteristic syndrome of sore throat and painful lymphadenopathy followed by a septicaemic illness with metastatic abscess formation should alert the clinician to the possibility of this unusual infection.
We express our thanks to Dr Bernstein and $\mathrm{Mr}$ Rahman for allowing us to present this case.

1 Lemierre A. On certain septicaemias due to anaerobic organisms. Lancet 1936;i:701-3.

Alston JM. Necrobacillosis in Great Britain. $\mathrm{Br}$ Med $J$ 1955;ii:1524-8.

3 Kleinman PK, Flowers RA. Necrotizing pneumonia after pharyngitis due to Fusobacterium necrophorum. Paediatr Radiol 1984;14:49-51.

4 Felner JM, Dowell VR. "Bacteroides" bacteremia. Am J Med 1971;50:787-95.

5 Seidenfeld SM, Sutker WL, Luby JP. Fusobacterium necrophorum septicaemia following oropharyngeal infection JAMA 1982;248:1348-50.

6 Moore-Gillon J, Lee TH, Eykyn SJ, Phillips I. Necrobacillosis: a forgotten disease. $\mathrm{Br}$ Med $J$ 1984;288:1526-7.

7 Oleske JM, Starr SE, Nahmias AJ. Complications of peritonsillar abscess due to Fusobacterium necrophorum. 4:570-1.

8 Vogel LC, Boyer KM. Metastatic complications of Fusobacterium necrophorum sepsis. Am J Dis Child $1980 ; 134: 356-8$ 